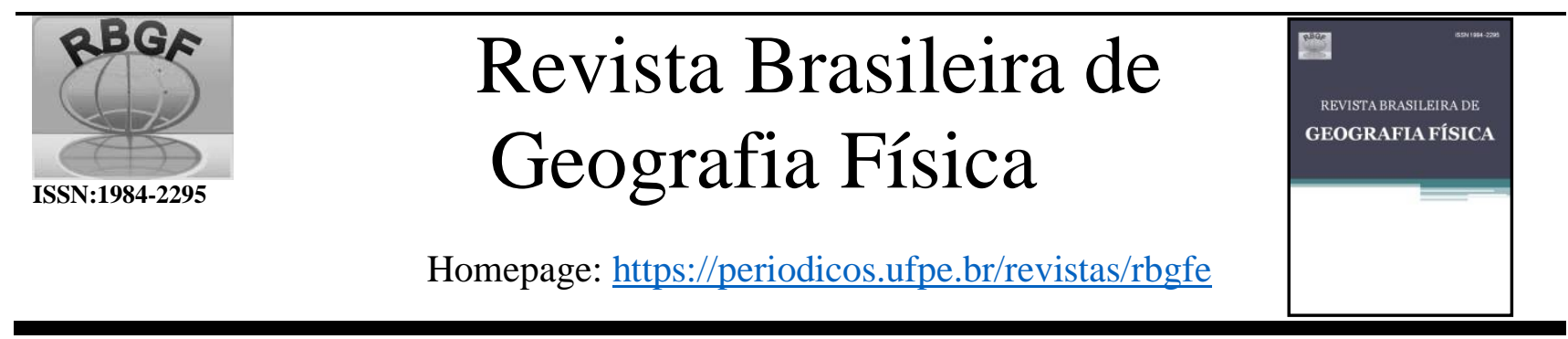

\title{
Uso de imagens tomadas por aeronaves remotamente pilotadas para detecção da cultura do milho infestada por Spodoptera frugiperda
}

\author{
Denner Borges Resende ${ }^{1}$, Carlos Alberto Matias de Abreu Júnior ${ }^{2}$, George Deroco Martins ${ }^{3}$, Odair José \\ Marques $^{4}$, Laura Cristina Moura Xavier ${ }^{5}$
}

\begin{abstract}
${ }^{1}$ Discente do curso de Agronomia, Instituto de Ciências Agrarias, Universidade Federal de Uberlândia, km 1, LMG-746, CEP 38500-000, Monte Carmelo, Minas Gerais. (34) 3810-1010. denner.borges@ hotmail.com (autor correspondente). ${ }^{2}$ Discente do curso de Engenharia de Agrimensura e Cartográfica, Instituto de Geografia, Universidade Federal de Uberlândia, km1, LMG-746, CEP 38500-000, Monte Carmelo, Minas Gerais. (34) 3810-1010. carlosalberto0103@ hotmail.com. ${ }^{3}$ Professor Dr., Instituto de Engenharia Civil, Universidade Federal de Uberlândia, km 1, LMG-746, CEP 38500-000, Monte Carmelo, Minas Gerais. (31) 3810-1010. deroco@ufu.br. ${ }^{4}$ Professor Dr., Instituto de Ciências Agrarias, Universidade Federal de Uberlândia, km 1, LMG-746, CEP 38500-000, Monte Carmelo, Minas Gerais. (34) 3810-1010. ojmarques@ ufu.br. ${ }^{5}$ Discente do curso de Engenharia de Agrimensura e Cartográfica, Instituto de Geografia, Universidade Federal de Uberlândia, km1, LMG-746, CEP 38500-000, Monte Carmelo, Minas Gerais. (34) 3810-1010. xavier.lauramoura@gmail.com.
\end{abstract}

Artigo recebido em 16/08/2019 e aceito em 01/03/2020

\section{RE S U M O}

A Spodoptera frugiperda (Smith) (lagarta-do-cartucho) é a principal praga do milho, com a intensificação da agricultura, os cultivos sucessivos possibilitam maior infestação pela praga. Isso levou ao surgimento de populações resistentes a inseticidas e culturas transgênicas. Pesquisas de campo para iniciar tratamentos com inseticidas são demoradas e exaustivas. Pensando em agilidade e qualidade, neste trabalho foi utilizado uma aeronave remotamente pilotada (ARP) equipado com uma câmera RGB e uma câmera MAPPIR 3 para capturar imagens de uma lavoura de milho, com o objetivo de estimar o índice de área foliar (IAF) de um talhão infestado por S. frugiperda. Durante o ciclo da cultura do milho foram realizadas várias avaliações: determinação do índice de área foliar (IAF), severidade do ataque da praga e, voos para aquisição das imagens. Modelos radiométricos para estimativa do IAF foram obtidos a partir de modelos de regressão linear compostos pelas bandas que melhor correlacionaram com os parâmetros medidos. Os resultados obtidos demonstraram eficiência e maior precisão na estimativa do IAF para o modelo radiométrico composto pela a banda do infravermelho próximo na câmera MAPPIR 3. Nesta ocasião, o RMSE calculado foi de $885,0714 \mathrm{~cm}^{2}$.

Palavras-chave: Milho; Lagarta-do-cartucho; Aeronave Remotamente Pilotada.

\section{Use of images for attack detection of Spodoptera frugiperda in corn under function of loss of foliar area}

\begin{abstract}
A B S T R A C T
Spodoptera frugiperda (Smith) (cartridge-caterpillar) is the main pest of maize, with the intensification of agriculture, the successive crops allow greater infestation by the pest. This has led to the emergence of populations resistant to insecticides and transgenic crops. Field research to begin treatments with insecticides is time consuming and exhaustive. Thus, thinking of agility and quality, in this work was used a remotely piloted aircraft (ARP) equipped with an RGB camera and a MAPPIR 3 camera to capture images of a maize crop with the objective of estimating the leaf area index (LAI) of a field infested by S. frugiperda. The radiometric models for the estimation of LAI were obtained from linear regression models composed by the bands that best correlated with the measured parameters. The obtained results demonstrated efficiency and greater accuracy in the estimation of the LAI for the radiometric model composed by the near infrared band (IVP) in the MAPPIR 3 camera. On this occasion, the RMSE calculated was $885.0714 \mathrm{~cm}^{2}$.
\end{abstract} Keywords: Corn; Cartridge Caterpillar; Remotely Piloted Aircraft.

\section{Introdução}

O uso exagerado ou não racional da tecnologia de milho híbrido geneticamente modificado com o gene $\mathrm{Bt}$ (Bacillus thuringiensis), para o controle de lepidópteros, sobretudo da $\mathrm{S}$. frugiperda, tem ocasionado a seleção de populações resistentes da praga (Bernardi et al., 2017). 
Os prejuízos causados pela $S$. frugiperda anualmente chegam na casa de milhões de dólares. Os danos provocados pela praga são mais expressivos a partir da fase de cartucho (estádio fenológico V8), consequentemente, afetando a produtividade final e diminuindo o índice de área foliar (IAF), o que ocasiona menor enchimento de grãos. Assim, novas ferramentas de monitoramento da praga se fazem necessárias, a fim de evitar o uso irracional de inseticidas sobrepostos à tecnologia Bt. Entre essas ferramentas, o uso de imagens aéreas, correlacionadas com características agronômicas das plantas pode contribuir para uma agricultura mais racional.

A agricultura de precisão considera exatamente esse cenário: a presença, distribuição e intensidade de uma condição específica que causa redução no rendimento da cultura agrícola devem ser identificadas e receber tratamento diferenciado (Mahlein, 2017). Nesse contexto, o monitoramento remoto da resposta da vegetação em condições de estresse é particularmente importante em agricultura de precisão, uma vez que os custos relacionados com a intensa amostragem ou manejo agrícola indiscriminado para toda área de cultivo podem ser minimizados pelo mapeamento prévio das áreas de ocorrência da $S$. frugiperda.

Assim, o uso direcionado dessa prática, como ferramentas de planejamento, controle e análise de dados agrícolas, prevê, então, o monitoramento das variações espaciais e temporais dos fatores que limitam a produção, orientando no processo de tomada de decisão, na aplicação de insumos em áreas específicas e no manejo diferenciado das culturas no campo de produção.

Os diferentes tipos de dados de sensoriamento remoto obtidos em multiescala para o monitoramento e mapeamento de culturas agrícolas infectadas por parasitas, encontram-se em fase de constante avanço. Isto ocorre devido à quantidade de dados obtidos por intermédio de plataformas terrestres, aeronaves e/ou plataformas orbitais, o que facilita o acesso a produtos radiométricos oriundos de fontes diversas (Candiago et al., 2015).

Várias abordagens têm sido avaliadas para a detecção de doenças sintomáticas e assintomáticas de plantas a partir de dados obtidos por sensoriamento remoto em multiescala, ambos baseados em espectroscopia (Martins e Galo, 2015; Ashourloo et al., 2016;) ou em imagem multiespectral e hiperespectral (Martins e Galo, 2014).
Especificamente, o uso de drones na agricultura tem focado em sensores baseados na espectroscopia de reflectância, após interação com diferentes superfícies de diferentes comprimentos de ondas do chamado espectro refletida, mais especificamente na área do visível e do infravermelho próximo. Como indicadores de estresses, os sensores se dividem entre sensores que detectam a transpiração e a redução da absorção de $\mathrm{CO} 2$ na planta ou na redução da fotossíntese (Jorge e Inamasu, 2014).

O padrão espectral da vegetação prove uma série de padrões para identificar o estádio fisiológico e de saúde das plantas. Em comprimentos de onda na região visível do espectro eletromagnético, refletância (medida proporcional da radiação refletida por um objeto) da vegetação está relacionada com os pigmentos fotossintéticos como a clorofila a clorofila b, xantofilas, antocianinas e carotenoides (Féret et al., 2017). Nas imagens podemos enxergar a banda do visível, porém, algumas respostas como, por exemplo, a resposta de stress nutricional, estruturas do dossel como folhas atacadas por pragas e doenças são melhores vistas pelo infravermelho próximo. Sensores dessa natureza são capturados geralmente por câmeras multiespectrais que trocam a banda do azul pela do infravermelho próximo, ou seja, assim as bandas são trocadas de azul, verde e vermelho, por verde, vermelho e infravermelho próximo, tendo essa configuração a possibilidade criar índices de vegetações que são possíveis avaliar partes o dossel de grandes lavouras.

Desse modo, o objetivo deste trabalho foi avaliar o potencial de imagens tomadas por ARPs (Aeronaves Remotamente Pilotadas) em discriminar distintos tratamentos inseticidas aplicados sobre o milho e estimar o índice de área foliar da cultura do milho em função de diferentes tipos de manejo e de danos causados pela Spodoptera frugiperda.

\section{Material e métodos}

O experimento foi instalado, no período da safrinha de 2018, na área experimental da Universidade Federal de Uberlândia, Campus Monte Carmelo. Na Figura 1, está representado o mapa de localização espacial da área de estudo, que pertence a região do Alto Paranaíba, Estado de Minas Gerais, apresenta altitude aproximada de 908 m. A região destacada por vermelho na imagem indica o local onde foi realizado $o$ experimento, sendo cada um dos blocos da plantação subdivididos para a aplicação de 
diversos tratamentos. $\mathrm{O}$ solo da área é textura argilosa. caracterizado como Latossolo Vermelho de

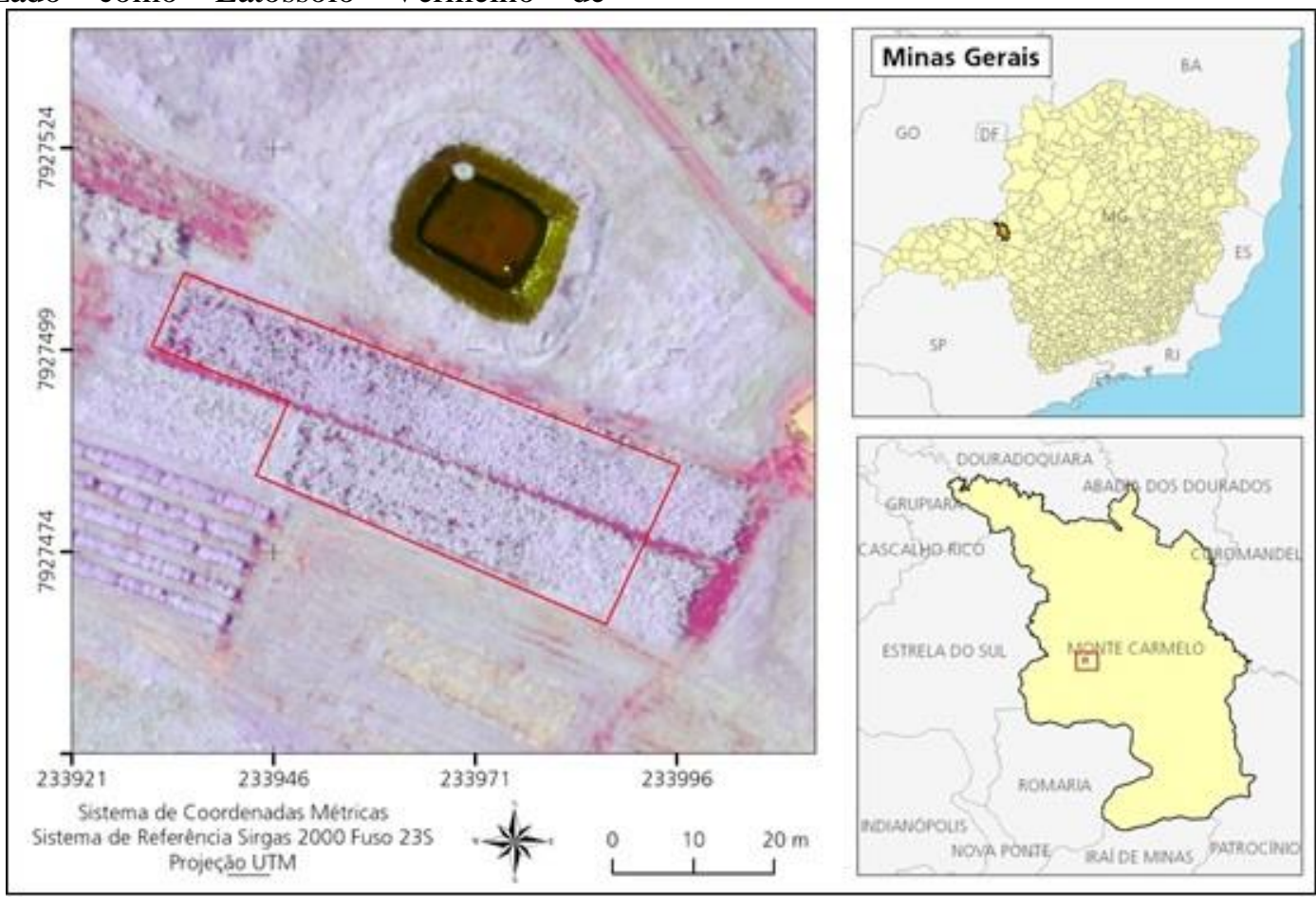

Figura 1. Mapa de Localização: Talhões de milho destacando a área de estudo em uma imagem MAPPIR 3 falsa cor.

O delineamento foi em blocos com tratamentos casualizados, contendo dois híbridos de milho (um convencional SHS4080® da Santa Helena Sementes e um transgênico Fórmula Viptera ${ }^{\circledR}$ da Syngenta Seeds) e seis tratamentos fitossanitários, descritos abaixo, com quatro repetições, totalizando em 48 parcelas. Cada parcela tem dimensões de $6,0 \times 2,0 \mathrm{~m}(12 \mathrm{~m} 2)$, contendo quatro linhas de plantas, sendo a área útil composta pelas duas linhas centrais com dimensões de 5,0 x 1,0 (5 m2).

Os tratamentos foram constituídos de: uma testemunha sem aplicação de inseticidas (apenas água), três inseticidas químicos e dois inseticidas biológicos, aplicados no mesmo dia, sempre ao entardecer/anoitecer, a saber: T1 (testemunha), $\mathrm{T} 2$ - bifentrina $180 \mathrm{~g}$ de ingrediente ativo (i.a.) L-1; zetacipermitrina $200 \mathrm{~g}$ i.a. L-1 (Hero®), dose de $200 \mathrm{~mL}$ do produto comercial (p.c.) ha-1, T3 - tebufenozide 240 g i.a. L-1 (Mimic $\left.{ }^{\circledR}\right)$, dose de $300 \mathrm{~mL}$ p.c. ha-1, T4 chlorfenapyr 240 g i.a. L-1, dose de $750 \mathrm{~mL}$ p.c. ha-1 (Pirate®), T5 - espinosade 480 g i.a. L-1 (Tracer®), dose de $100 \mathrm{~mL}$ p.c. ha-1 e T6 lambda-cialotrina $250 \mathrm{~g}$ i.a. L-1 (Kaiso 250CS®), dose de $30 \mathrm{~mL}$ p.c. ha-1. A distribuição destes tratamentos está apresentada na Figura 2.

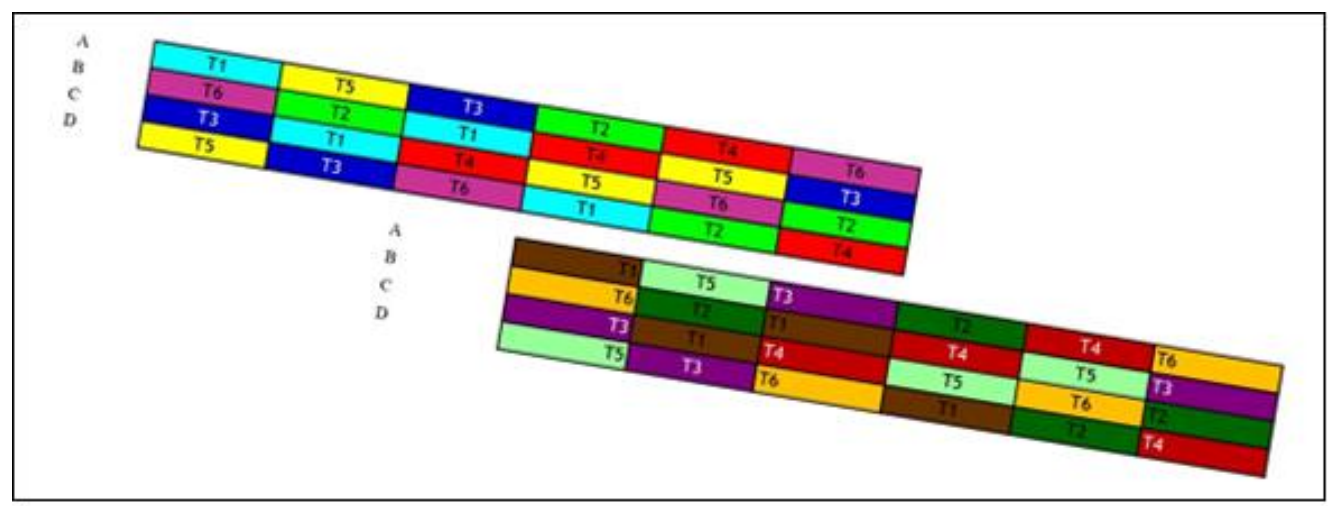

Figura 2. Distribuição dos tratamentos na área de estudo.

Todos os produtos foram preparados em taxa de aplicação equivalentes para $200 \mathrm{~L}$ de calda 
por ha, sendo a aplicação realizada com auxílio de um cilindro de $\mathrm{CO} 2$ e uma barra com quatro pontas de pulverização, totalizando uma faixa de aplicação de $1,5 \mathrm{~m}$ de largura, de modo que as duas linhas centrais receberam os tratamentos em dose cheia.

As aplicações foram realizadas logo após a primeira avaliação de danos da praga (estádio V3), em que foi observado o ataque da $S$. frugiperda, como raspagem e furos de acordo com a metodologia de avaliação de Davis \& William (1989a, 1989b), sendo que o controle foi realizado ao se atribuir a nota 2 ou superior em qualquer uma das parcelas. A partir da primeira aplicação dos tratamentos fitossanitários, a escala de Davis foi utilizada apenas para verificação da eficiência dos tratamentos.

Durante o ciclo da cultura foram realizados todos os tratos culturais exigidos pela cultura do milho.

Mensurou-se a área foliar (AF) logo após a constatação dos estádios V4, V10 e VT. Para tanto, foram avaliadas cinco plantas ao acaso em cada parcela, sendo mensurado o comprimento total e a largura do meio da folha, em centímetros, de todas as folhas de cada uma das plantas. Para o cálculo da AF, foi empregada a equação 1, proposta por Francis (1969):

$$
\mathrm{AF}=0,75^{*}\left(\mathrm{C} / 100^{*} \mathrm{~L} / 100\right)
$$

onde: $\mathrm{AF}$ - área foliar $\mathrm{m} 2$; $\mathrm{C}$ comprimento da folha $\mathrm{cm}$; L - largura da folha $\mathrm{cm}$.

Em seguida, o IAF será calculado a partir das medidas de $\mathrm{AF}$, conforme a equação 2 :

$$
I A F=(\mathrm{AF} /(\mathrm{e} 1 * \mathrm{e} 2))
$$

onde: IAF - índice de área foliar; e1 espaçamento entre plantas $\mathrm{m}$; e2 - espaçamento entre linhas de plantas $m$.

Após a determinação da data da primeira aplicação dos tratamentos fitossanitários, as plantas (no estádio V4) foram monitoradas no $7^{\circ}$ dia após a aplicação (DAA1), em seguida após a segunda aplicação o monitoramento aconteceu no $7^{\circ}$ e $15^{\circ}$ (DAA2), respectivamente nos estádios V5 e V8.

O monitoramento consistiu da avaliação de cinco plantas por parcela, atribuindo a cada planta notas na escala de Davis, sendo utilizada para verificar severidade de ataque, sendo o resultado final a razão da somatória das notas pelo número de plantas avaliadas em cada parcela. As coordenadas geográficas de cada planta avaliada foram georreferenciadas para a correlação das variáveis medidas in situ, com os valores de reflectância da imagem obtida por drone.

Concomitante as medidas do IAF, as imagens multiespectrais foram adquiridas a partir de uma câmera multiespectral MAPIR 3, contendo as bandas do intervalo espectral médio do verde $(550 \mathrm{~nm})$, vermelho $(660 \mathrm{~nm})$ e infravermelho próximo $(850 \mathrm{~nm})$.

A câmera foi instalada na posição nadir em um ARP, drone Phantom 4 Pro e, o voo foi realizado às $12 \mathrm{~h}$ com uma altitude de $165 \mathrm{~m}$ e uma configuração da resolução espacial das imagens em 12,5 cm. No momento da aquisição de imagens, foram instaladas na superfície próxima do talhão, placas de papel fotográfico nas cores: azul, verde, vermelho, branco e preto para a posterior calibração radiométricas das imagens adquiridas. Na Figura 3 é apresentado o sistema de aquisição de imagens: drone Phantom 4 Pro e Câmara MAPIR 3.

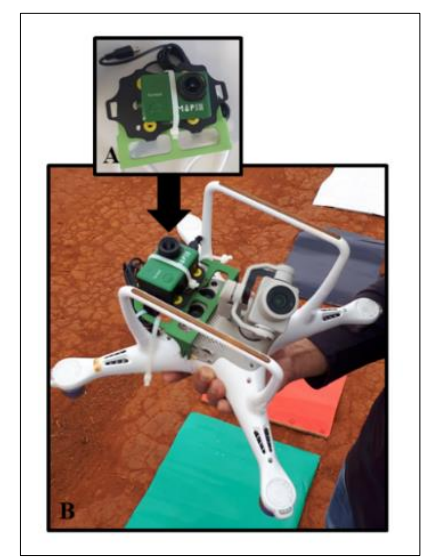

Figura 3. Drone Phantom 4 Pro e câmera MAPIR 3. (A) Suporte com a câmara MAPIR 3. (B) Câmara MAPIR 3 acoplada ao drone Phantom 4 Pro.

O mosaico de imagens foi gerado no software Pixel4D. Na etapa de fototriangulação, não foram utilizados pontos de apoio materializados no terreno, para formação do mosaico, apenas utilizou-se as coordenadas geográficas do centro perspectivo das imagens e os ângulos de orientação exterior proveniente de um sistema inercial embaracado na própria câmera MAPIR 3. A calibração radiométrica do mosaico foi realizada de forma automática com o software Mapir Camera Control.

Para analisar o potencial das imagens em discriminar os distintos tipos de tratamento inseticidas para os diferentes híbridos de milho em função da radiometria do dossel do experimento, a partir de uma amostra de 2000 pontos representativos para cada um dos tratamentos, foram gerados gráficos de distribuição de frequência, análise de agrupamentos, cálculo da média e desvio padrão. 
$\mathrm{Na}$ análise de agrupamento, foram gerados os possíveis clusters considerando um nível de similaridade de $68 \%$. Ao final da análise estatística, foi gerado um mapa de distribuição de classes de informação, no qual cada classe referiuse aos 12 tratamentos inseticidas aplicados sobre o experimento. Para geração do mapa, foi aplicada a classificação supervisionada do tipo Máxima Verossimelhança, considerando uma similaridade $99 \%$.

A imagem foi classificada considerandose como arquivo de classificação a associação das três bandas originais da câmara MAPIR 3 com o índice NDVI. O mesmo conjunto amostral de 2000 pontos utilizados nas análises estatísticas foi utilizado para o treinamento do algoritmo de classificação.

Ressalta-se que nesta etapa, os tratamentos estão codificados conforme: Tx_y, onde $\mathrm{T}$ significa tratamento, $\mathrm{x}$ significa $\mathrm{um}$ identificador variável de 1 a 6 , indicando o tipo de tratamento e y significa um identificador variável de 1 a 2, indicando os dois híbridos do milho: 1convencional e 2- transgênico.

Para geração dos modelos empíricos, primeiramente, foram avaliadas a correlação Pearson entre o IAF e as bandas originais da câmera MAPIR 3.

O critério para escolha das bandas e dos índices de vegetação para composição dos modelos foi definido a partir dos canais que apresentaram uma significativa e maior correlação com o IAF. Deste modo, definiu-se como significativa à correlação superior a $50 \%$ e um nível de significância de 5\%.

Os modelos predição foram definidos a partir de equações de regressão lineares simples entre valores radiométricos medidos na imagem e a concentração do IAF. Para tanto se considerou a radiância e o IAF de 180 amostras (definidas aleatoriamente) entre os 210 pontos de coletas contidos dentro da área de estudo.
A validação da precisão dos modelos de predição foi realizada estimando-se o IAF em 30 (definidos aleatoriamente) dos 210 pontos amostrados in situ. A partir dos dados de discrepância entre o IAF observado e estimado, foi calculado o erro médio quadrático (RMSE) da amostra. O RMSE e erro (RMSE (\%)) foi calculado a partir do modelo da equação 3 :

$$
R M S E=\sqrt{\frac{\sum_{i=1}^{n}\left(x_{i}-x_{m e d}\right)^{2}}{n}}
$$

onde: xi e xmed - valor estimado e medido in situ do TCT, respectivamente; $\mathrm{n} \mathrm{-}$ número de amostras.

Além da análise da acurácia, a tendência do modelo gerado foi avaliada a partir de um teste de hipótese $\mathrm{t}$ de Student, onde as hipóteses formuladas foram: H0: Tendência $=0$ e H1: Tendência $\neq 0$. $O$ teste $t$ de Student foi calculado a partir da equação 4 :

$$
T=\frac{X_{n}-\mu}{\frac{S_{n}}{\sqrt{v}}}
$$

onde: Xn: média populacional; $\mu$ referência; Sn - desvio padrão amostral; v - graus de liberdade.

Ao final, com o modelo empírico foi gerada uma carta contendo o mapeamento do IAF sobre os talhões milho. O mapa coroplético foi divido em cinco classes em intervalos iguais, apresentando a magnitude do IAF em ordem crescente de cinco unidades de valores de medida. Deve-se ressaltar que dentre possíveis métodos de definição de classes de valores para a carta gerada, o método utilizado foi o fatiamento por intervalos iguais.

\section{Resultados e discussão}

A Figura 4 apresenta os dendrogramas gerados a partir da análise de similaridade dos números digitais correspondentes aos distintos tratamentos inseticidas observados nas bandas do verde, vermelho, infravermelho próximo e o índice NDVI. 


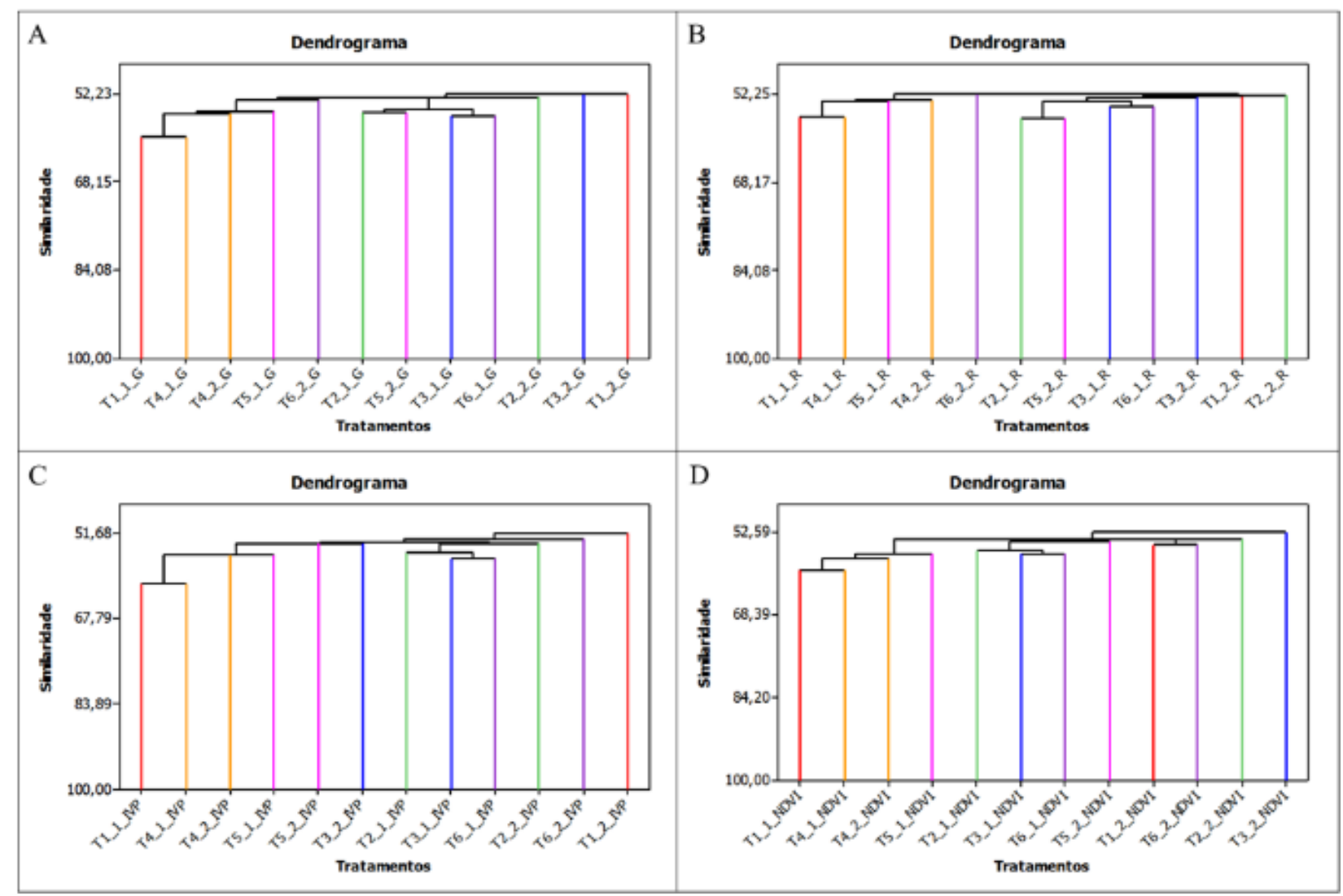

Figura 4. Dendograma discriminando à similaridade entre os tratamentos para as bandas originais e os índices derivados.

Ao analisar os dendrogramas gerados observa-se em cada um a formação de 12 agrupamentos distintos, ou seja, a partir de todas as bandas e do índice NDVI é possível discriminar os todos os tipos de tratamentos para os dois híbridos de milho, considerando valores inferiores um nível de similaridade de 1 sigma (68\%).
A Figura 5 apresenta a distribuição de frequência dos números digitais para referente às amostras de treinamento para cada tratamento. Gráficos: A-números digitais da banda do verde, B- números digitais da banda do vermelho, Cnúmeros digitais da banda do infravermelho próximo e D- números digitais do índice NDVI. 


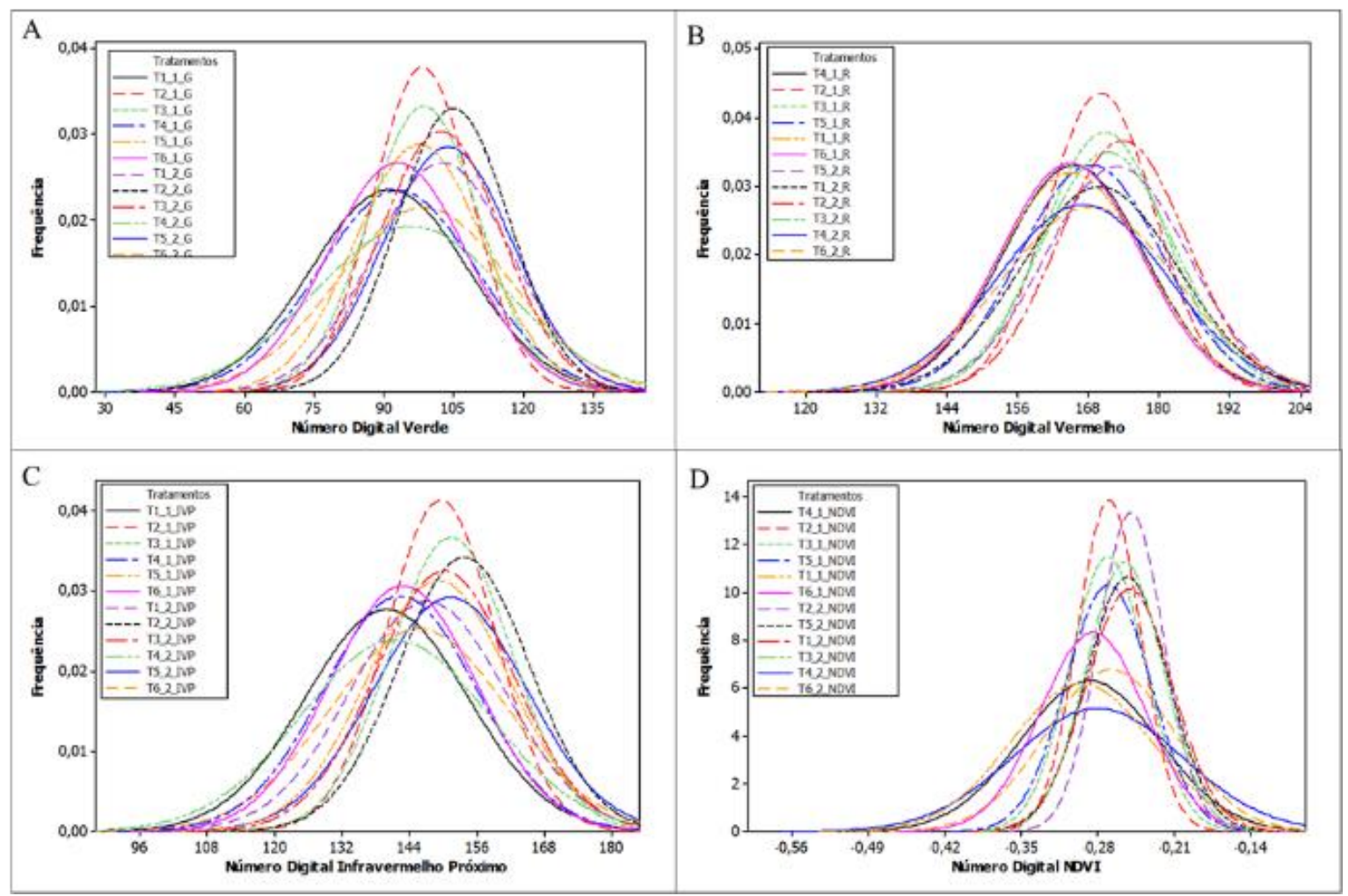

Figura 5. Diagrama de frequência para cada um dos tratamentos. A-números digitais da banda do verde, Bnúmeros digitais da banda do vermelho, $\mathrm{C}$ - números digitais da banda do infravermelho próximo e Dnúmeros digitais do índice NDVI.

A partir da Figura 5 observa-se que para todas as amostras de treinamento, a distribuição de frequência para cada um dos tratamentos segue um padrão de normalidade. Esta condição permite que em processos de discriminação de tratamentos por imagem, possam ser aplicados classificadores supervisionados estatísticos, tais como o de máxima verossimilhança (Martins et al., 2017). Porém, em processos desta natureza observa-se que para classes de informação deste estudo, não podem ser discriminadas com critério de semelhança inferior a 99\%, uma vez que a há pouca aderência entre as médias e a amplitude dos diferentes dados de treinamento para cada uma das bandas e índice de vegetação analisado.

Neste experimento, a proximidade do valor médio da resposta espectral para todas as amostras de treinamento pode ser justificada pelo fato de que o vigor vegetativo das distintas parcelas tenha sido pouco ataque da S. frugiperda, ou seja, a cor das folhas e a taxa de fotossíntese das áreas atacadas pouco diferenciou das áreas sadias. Assim, para discriminar os diferentes tipos de tratamento, a partir da Figura 5, observa-se que uma única banda não é possível discriminar tais classes de informação, mas sim, a partir da associação das mesmas com o índice de vegetação NDVI.
Para uma análise mais pontual da resposta espectral dos distintos tratamentos, na Tabela 1 são apresentados os valores de média e desvio padrão do número digital das bandas e o índice NDVI para cada uma das classes de informação.

Tabela 1. Estatística descritiva de média e desvio padrão dos tratamentos. Colunas: 1Identificação do tratamento; 2-Número digital na banda do verde; 3- Número digital na banda do vermelho; 4- Número digital na banda do infravermelho próximo; 5- Número do índice NDVI.

\begin{tabular}{|c|c|c|c|c|}
\hline $\begin{array}{c}\text { Híbrido 1 } \\
\text { Convenci. }\end{array}$ & $\begin{array}{c}\text { Média/ } \\
\text { Desvio } \\
\text { Padrão } \\
\text { (verde) }\end{array}$ & $\begin{array}{c}\text { Média/ } \\
\text { Desvio Padrão } \\
\text { (vermelho) }\end{array}$ & $\begin{array}{c}\text { Média/ Desvio } \\
\text { Padrão (IVP) }\end{array}$ & $\begin{array}{c}\text { Média/ Desvio } \\
\text { Padrão (NDVI) }\end{array}$ \\
\hline 1 & $165,05 / 0,280$ & $91,176 / 0,379$ & $139,91 / 0,322$ & $0,2947 / 0,00144$ \\
\hline 2 & $170,07 / 0,205$ & $98,278 / 0,236$ & $149,50 / 0,216$ & $0,2694 / 0,00064$ \\
\hline 3 & $170,73 / 0,236$ & $98,584 / 0,268$ & $151,38 / 0,243$ & $0,2705 / 0,00077$ \\
\hline 4 & $165,49 / 0,270$ & $92,968 / 0,380$ & $142,29 / 0,304$ & $0,2871 / 0,00142$ \\
\hline 5 & $168,45 / 0,270$ & $97,557 / 0,310$ & $149,07 / 0,285$ & $0,2701 / 0,00086$ \\
\hline 6 & $164,95 / 0,268$ & $92,894 / 0,335$ & $142,79 / 0,291$ & $0,2841 / 0,00107$ \\
\hline Híbrido 2 & $\begin{array}{c}\text { Média/ } \\
\text { Desvio }\end{array}$ & $\begin{array}{c}\text { Média/ } \\
\text { Padrão } \\
\text { (verde) }\end{array}$ & $\begin{array}{c}\text { Média/ Desvio } \\
\text { (vermelho) }\end{array}$ & Média/ Desvio \\
\hline 1 & $169,86 / 0,298$ & $102,54 / 0,334$ & $147,17 / 0,311$ & $0,2508 / 0,00088$ \\
\hline 2 & $173,82 / 0,244$ & $104,93 / 0,271$ & $153,68 / 0,261$ & $0,2493 / 0,00066$ \\
\hline 3 & $171,24 / 0,255$ & $102,26 / 0,295$ & $150,12 / 0,275$ & $0,2551 / 0,00079$ \\
\hline 4 & $167,05 / 0,327$ & $95,815 / 0,463$ & $141,39 / 0,374$ & $0,2800 / 0,0017$ \\
\hline 5 & $172,84 / 0,272$ & $103,78 / 0,313$ & $151,21 / 0,306$ & $0,2529 / 0,00083$ \\
\hline 6 & $168,20 / 0,331$ & $98,525 / 0,415$ & $145,65 / 0,353$ & $0,2680 / 0,00132$ \\
\hline
\end{tabular}

A partir da Tabela 1, observa-se que os maiores valores estão concentrados na banda do 
verde e não na banda do infravermelho próximo, onde há uma maior resposta e espalhamento da energia refletida em função do processo de fotossíntese (Martins et al., 2015). Esse comportamento deve-se ao fato da banda do infravermelho próximo estar centrada no início do intervalo de frequência da região termal, ou seja, $850 \mathrm{~nm}$, região onde a energia refletida da vegetação, ainda não é máxima (Martins et al., 2017).

Outra possibilidade que justifica os valores da resposta na banda infravermelho próximo menor do que na banda vermelho, é o fato de que sensores termais de baixo custo, como ao utilizado nesta pesquisa, são originalmente câmaras convencionais e só são capazes de capitar a energia refletida na região do termal, pois a banda do azul é substituída pela a do infravermelho próximo. $\mathrm{Na}$ maioria destes sensores, a banda do azul é a mais sensível à energia refletida na região do termal, assim esta técnica é possível a partir da aplicação de um filtro colocado sobre lente da câmera onde ocorre o bloqueio da leitura do comprimento de onda do azul, liberando apenas a captura de uma pequena magnitude de energia refletida na região do infravermelho próximo (Imai et al., 2017).

Em geral, observa-se que para as a três bandas, os dados sinalizam uma baixa variabilidade em torna das médias, onde os valores de número digital foram maiores para os tratamentos aplicados para o híbrido 2 (transgênico). Outro ponto observado é em relação aos baixos valores do índice NDVI, os quais são em maioria menores para os tratamentos aplicados para o híbrido 2, esta condição pode estar associada à influência da resposta do solo em áreas com baixo IAF resultante da desfolha do milho causada pelo ataque da S. frugiperda.

A Figura 6 apresenta um mapa de distribuição de classes de tratamento gerado a partir da classificação supervisionada de máxima verossimilhança.

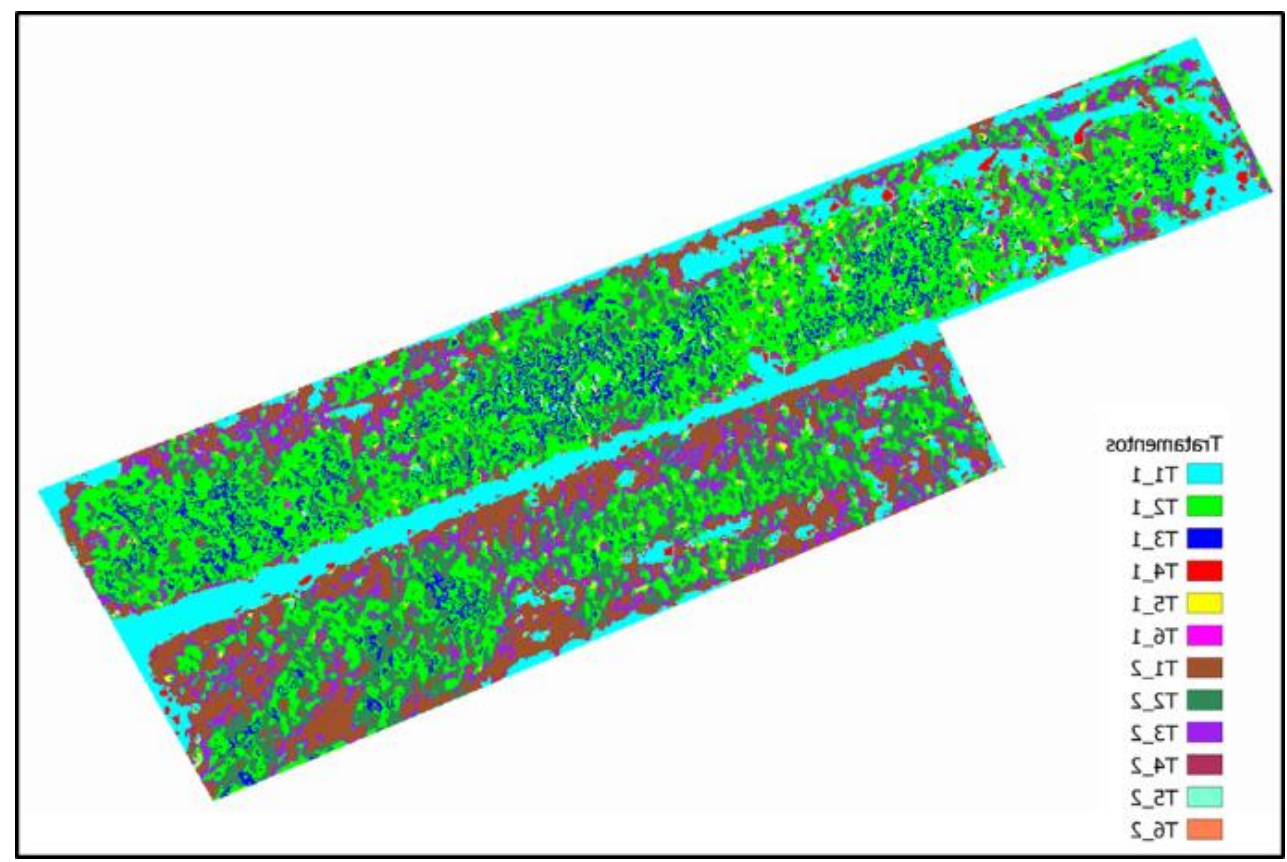

Figura 6. Mapa de distribuição de classes máxima verossimilhança dos tratamentos inseticidas.

A partir do mapa da Figura 6 observa-se que as classes de informações foram mapeadas de forma não homogênea ao longo de todo o experimento, ou seja, não há foi possível discriminar os distintos tratamentos inseticidas distribuídos espacialmente em parcelas retangulares de $12 \mathrm{~m}^{2}(6 \mathrm{~m} \times 2 \mathrm{~m})$. Verifica-se que houve um predomínio das classes referentes aos tratamentos T2_1 e T1_2 e que a classe T1_1 fora classificada em áreas onde havia alta resposta do solo exposto: 1- carreadores e 2-falhas causadas pelo ataque da $S$. frugiperda.

A Tabela 2 apresenta o valor da correlação de Pearson entre as bandas da câmera MAPPIR 3 e o IAF, na coluna 1, e respectivo Pvalor na coluna 3 .

Tabela 2. Coeficiente de correlação de Pearson e $\mathrm{P}$-valor entre as bandas da imagem e o IAF.

\begin{tabular}{|l|l|l|}
\hline Banda & IAF & P-valor \\
\hline $\begin{array}{l}\text { Infravermelho } \\
\text { próximo }\end{array}$ & 0,550 & 0,001 \\
\hline Verde & 0,545 & 0,001 \\
\hline
\end{tabular}




\begin{tabular}{|l|l|l|}
\hline Vermelho & 0,516 & 0,003 \\
\hline NDVI & 0,530 & 0,002 \\
\hline
\end{tabular}

A partir da Tabela 2, observa-se que todas as correlações obtidas foram significativas, ou seja, para todas as relações o P-valor foi inferior a 0,005 . A radiância das bandas extraídas de pontos específicos mostrou melhores índices de correlação com a banda espectral do verde, 0,545, e maior correlação com a banda do infarvermelho próximo, 0,550. A banda do vermelho apresentou a menor correlação com o IAF, 0,516.

A maior correlação com o intervalo do infravermelho próximo é influenciada pela estrutura celular do mesófilo, no qual ocorre elevado espalhamento da radiação. Dessa forma, mudanças estruturas no tecido e na área foliar devido a variações no suprimento e deficiência nutricional, a exemplo da disponibilidade de $\mathrm{N}$ tem efeito considerável na resposta nessa faixa. Já as bandas do verde e do vermelho são altamente sensíveis a mudanças na concentração de clorofila, sendo o nitrogênio o principal elemento que induz essas alterações, assim espera-se que para esses intervalos, a correlação seja maior para medidas correlacionadas a clorofila.

Em estudos como de Tumlisan (2017), a partir de imagens capturadas por ARPs, também foi observada a possibilidade de correlacionar a banda do infravermelho próximo com o grau de dano causado por doenças no milho. Neste estudo, o autor ressalta a importância da banda no comprimento de onda no termal para detecção de injúrias foliares que ainda não estejam visíveis em situações de inspeções visuais de campo.

De fato, em diversas culturas, as bandas localizadas no intervalo do termal e os índices de vegetação contribuem para detecção de parâmetros e atributos agronômicos relacionados à presença de doenças (Martins et al., 2017), porém deve-se ressaltar que a alta resolução espacial de bandas centradas nos intervalos do visível também contribuir no monitoramento de parâmetros físicos (IAF) de culturas com folhas grandes como a do milho (Raeva et al., 2018).

A parir da relação entre a radiância da banda do infravermelho próximo e o IAF, o modelo de predição do IAF é descrito a partir da equação 5 .

$$
\mathrm{IAF}=-7369+\mathrm{IVP} * 69
$$

onde: IAF - índice de área foliar; IVP valores de radiância do infravermelho próximo.

Dada a utilização de uma única variável para estimativa de IAF, a equação 5 apresentou um coeficiente determinístico relativamente baixo na ordem de $26,6 \%$. Deve-se ressaltar que o uso de mais bandas ou a associação do índice NDVI poderiam resultar em um modelo de estimativa mais robusta por técnicas de regressão múltipla, porém dada a alta correção entre as bandas e o índice, a técnica de regressão múltipla torna-se inviável devido a colinearidade entre as variáveis preditoras.

Além do mais, apesar da função resposta do ter sido uma função linear, alguns pontos devem ser ressaltados, primeiramente, esta função só será válida para este estágio de desenvolvimento da cultura, V8, e para as condições de manejo em que a cultura fora submetida. Além do mais, geralmente, na cultura do milho, o índice de área foliar é sensível a variações temporais, onde, esta variação pode ser descrita por uma função quadrática. Há um decréscimo devido à senescência e queda das folhas logo após ser atingido um ponto de máximo. A intensidade destas variações são reflexos da variabilidade temporal da partição de foto assimilados que está relacionada à temperatura, assumindo que os demais fatores de produção sejam constantes.

A Figura 7 apresenta o RMSE do modelo de estimativa da IAF.

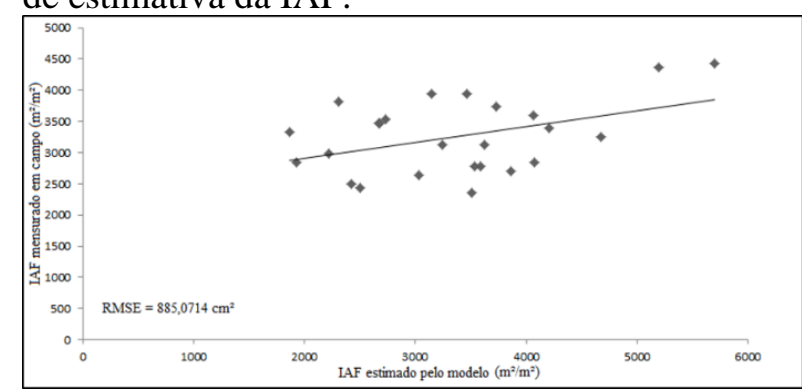

Figura 7. RMSE para a variação do erro ao longo das amostras (equação 5).

O gráfico de RMSE da Figura 7 apresenta os pontos plotados referentes aos valores mensurados em campo de IAF, no eixo das ordenadas, e valores estimados de IAF pelo modelo, no eixo das abcissas. A reta do gráfico transcreve o comportamento das variações entre cada valor, calculado e mensurado em campo. O RMSE obtido do foi de $885,0714 \mathrm{~cm}^{2}$ para uma amostra de validação com o coeficiente determinístico de $25,1 \%$. Nesta condição, este erro é equivalente a uma folha de $8 \mathrm{~cm}$ de largura por $100 \mathrm{~cm}$ de comprimento. Dessa forma, no que tange a aplicabilidade da fórmula, os resultados apresentaram valores satisfatórios, e condizentes com o determinado em campo, constatando a validade do modelo.

Segundo Ponzoni et al. (2012), alguns modelos provenientes de sensores como o MODIS 
são concebidos para realizar estimativas de IAF na escala diária, enquanto outros simulam a partir da média de um período mais longo, como um estádio fenológico ou o ciclo da cultura. De uma maneira ou de outra, o conhecimento da ordem de grandeza do índice de área foliar é imprescindível para estimativa da produtividade da cultura de milho e, é natural que os modelos tenham um uma acurácia inferior a $50 \%$.

Outro ponto quanta contribui para diminuir a acurácia dos modelos de estimativa de variáveis agrícolas é alta resolução espacial das imagens adquiridas por sensores desta natureza. Nesta perspectiva há uma dificuldade de se relacionar o pixel da imagem que representa com exatidão a posição exata da folha teve as variáveis agrícolas mensuradas em campo. Neste contexto, a imprecisão entre os receptores GNSS embarcado na ARP e utilizados nas medidas in situ, também, contribuem para inexatidão do modelo.

A figura 8 apresenta o mapa de distribuição espacial dos valores de IAF calculados.

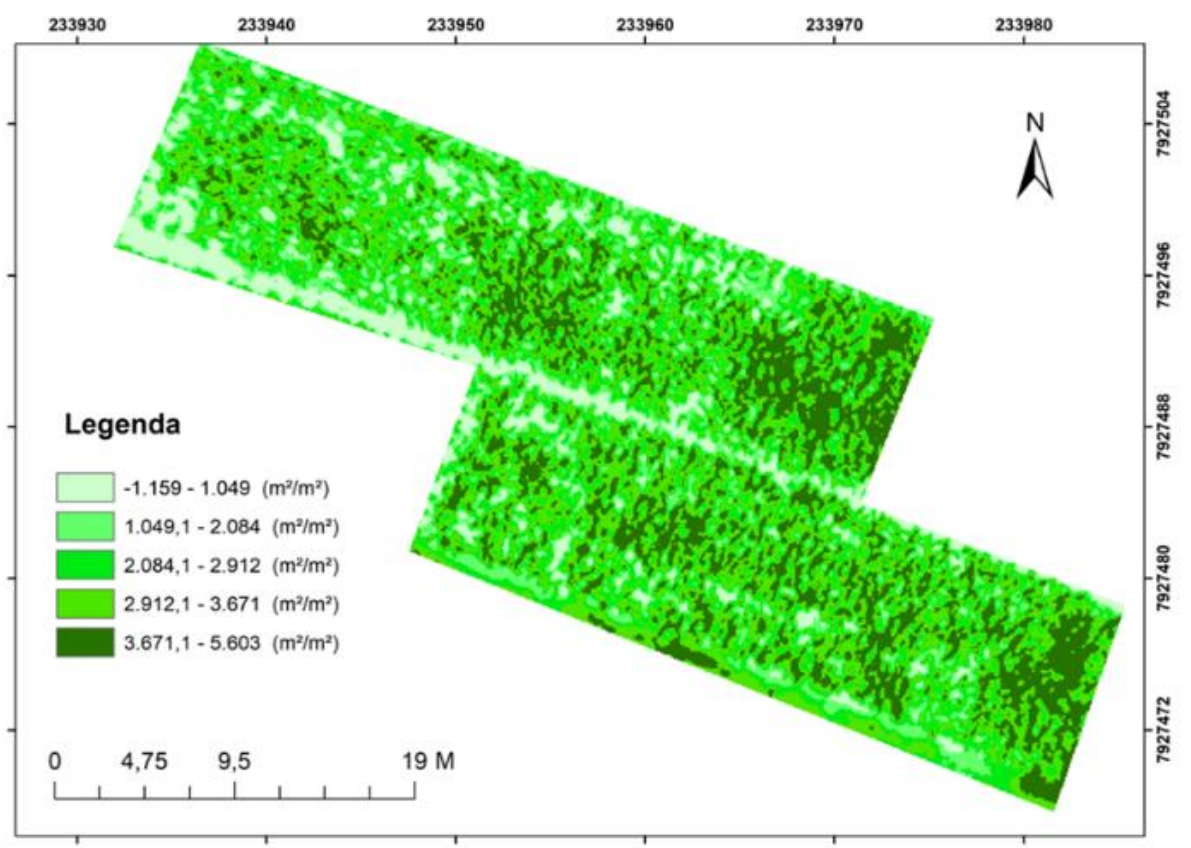

Figura 8. Mapa de IAF gerado através da equação de regressão linear em função do infravermelho próximo.

A partir da carta da Figura 8, observa-se que ao longo dos dois talhões há dispersão de diversos pontos com máxima concentração de IAF, sendo que a maior concentração se encontra à direita dos dois talhões. As regiões onde se evidenciam a menor concentração de IAF referem-se justamente as áreas onde foram encontrados focos $S$ frugiperda. Em uma visão geral, a carta evidencia-se que a distribuição do IAF é abrupta e não contínua, a este fato pode-se associar os diferentes tipos de manejos aplicados as distintas parcelas.

Os valores negativos no mapa são referentes a "ruídos" na imagem, e não ao valor espectral das plantas. Para Danelichen et al. (2016) que estima o IAF através de imagens orbitais, esses ruídos são um dos fatores que provavelmente influenciam na diferença encontrada entre o cálculo do índice com dados mensurados em campo e em modelos.

\section{Conclusão}

A partir da análise de números digitais, foi possível discriminar os diferentes tratamentos inseticidas aplicados sobre os dois híbridos do milho com um nível de significância de 68\%, porém o mapa de distribuição espacial dos tratamentos evidenciou classes de informação dispostas de forma não equivalentes às parcelas de tratamento.

Foi possível observar a perda do Índice de Área Foliar por tratamentos e assim servindo como embasamento para tomada de decisões. O modelo gerado a partir da equação do Índice de Área Foliar apresentou bons resultados, pois as plantas com injurias apresentaram uma resposta espectral menor que as plantas saudáveis, o que interfere diretamente no valor de Índice de Área Foliar.

Obteve-se uma correlação satisfatória entre o Índice de Área Foliar e as imagens aerofotogramétricas. O método estabelecido é 
eficiente para o monitoramento dos danos da Spodoptera frugiperda em lavoura de milho.

\section{Agradecimentos}

Os autores agradecem o apoio da

Universidade Federal de Uberlândia e também a MR AGROPESQUISA.

\section{Referências}

Ashourloo, D., Matkan, A.A., Huete, A., Aghighi, H., Mobasheri, M.R., 2016. Developing an index for detection and identification of disease stages. IEEE Geoscience and Remote Sensing Letters 13, 851-855.

Bernardi, D., Bernardi, O., Horikoshi, R.J., Salmeron, E., Okuma, D.M., Farias, J.R., Omoto, C., 2017. Selection and characterization of Spodoptera frugiperda (Lepidoptera: Noctuidae) resistance to MON 89034× TC1507× NK603 maize technology. Crop protection 94, 64-68.

Candiago, S., Remondino, F., Giglio, M., Dubbini, M., Gattelli, M., 2015. Evaluating multispectral images and vegetation indices for precision farming applications from UAV images. Remote sensing 7, 4026-4047.

Danelichen, V.H.M., Biudes, M.S., Machado, N.G., Velasque, M.C.S., Silva, B.B., Nogueira, J.S. 2016. Uso do sensoriamento remoto na estimativa do índice de área foliar em floresta tropical. Ciência e Natureza 38, 1352-1360.

Davis, F. M.; Williams, W. P. Methods used to screen maize for and to determine mechanisms of resistance to the southwestern corn borer and fall armyworm. In: International Symposium On Methodologies For Developing Host Plant Resistance To Maize Insect, 1989, México. Proceedings... México: [s.n.], 1989. p. 101-108.

Féret, J.B., Gitelson, A.A., Noble, S.D., Jacquemoud, S., 2017. PROSPECT-D: towards modeling leaf optical properties through a complete lifecycle. Remote Sensing of Environment 193, 204-215.
Imai, N.N., Moriya, E.A.S., Honkavaara, E., Miyoshi, G.T., Moraes, M.V.A., Tommaselli, A.M.G., Näsi, R., 2017. Analysis of the radiometric response of orange tree crown in hyperspectral uav images. Isprs - International Archives of the Photogrammetry, Remote Sensing and Spatial Information Sciences 42, 73-79.

Jorge, L.A.C., Inamasu, R.Y., 2014. Uso de veículos aéreos não tripulados (VANT) em agricultura de precisão. Agricultura de Precisão 1, 109-134.

Mahlein, A. K., 2016. Plant disease detection by imaging sensors-parallels and specific demands for precision agriculture and plant phenotyping. Plant disease 100, 241-251.

Martins, G.D., Galo, M.L.B.T., 2014. Detecção de áreas infestadas por nematoides e Migdolus fryanus em cultura canavieira a partir de imagens multiespectrais Rapideye. Revista Brasileira de Cartografia 66, 285-301.

Martins, G.D., Galo, M.L.B.T., 2015. Caracterização espectral da cana-de-açúcar infectada por nematoides e Migdolus fryanus por espectrorradiometria de campo. Bolem de Ciências Geodésicas 21, 783-796.

Martins, G.D., Galo, M.L.B.T., Vieira, B.S., 2017. Detecting and mapping root-knot nematode infection in coffee crop using remote sensing measurements. IEEE Journal of Selected Topics in Applied Earth Observations and Remote Sensing 10, 5395-5403.

Ponzoni, F.J., Shimabukuro, Y.E., Kuplich, T.M. 2012. Sensoriamento remoto da vegetação, 2 ed. Oficina de Textos, São Paulo.

Raeva, P.L., Edina, J., Dlesk, A., 2018. Monitoring of crop fields using multispectral and thermal imagery from UAV. European Journal Of Remote Sensing 52, 192-201.

Tumlisan, G.Y., 2017. Monitoring growth development and yield estimation of maize using very high-resolution UAV-images in Gronau, Germany. Thesis (Master). Enschede, University of Twente. 